\title{
Premature Ventricular Contraction Arrhythmia Detection Using Wavelet Coefficients
}

\author{
Asst Prof R.Himabindu, M.Tech,* Asst Prof M.Jyothirmai, M.Tech,** \\ B.Sulochana, (M.Tech), ${ }^{* * *}$ \\ *(Department of Electronics \& Communication Engineering, Ravindra engineering college for women, \\ Kurnool) \\ ** (Department of Electronics \& Communication Engineering, Ravindra engineering college for women, \\ Kurnool) \\ ***(Department of Electronics \& Communication Engineering, GPCET, and Kurnool)
}

\begin{abstract}
Premature ventricular contraction $(P V C)$ detection is an important task in critical care medicine. However, making this task automatic is not that simple. In this paper, we are describing a method for PVC arrhythmia detection. This method is based on the use of wavelet detail coefficients to discriminate between normal beats and abnormal beats (PVCS). The proposed method was tested against selected records of the MITBIH Arrhythmia Database (MITDB). Results are very satisfactory and show that it is possible to detect PVC arrhythmia using wavelet detail coefficients applied to QRS complexes.
\end{abstract}

\section{Introduction}

Arrhythmia detection is an important task in critical care medicine. Actually, the accurate and early detection of the different types of arrhythmia can prevent of dangerous heart problems. Premature ventricular contraction (PVC) is one of these arrhythmias. Many researches focused on the problem of automatic detection of PVC arrhythmia [1,2,3] and this research area is still active today due the difficulty to find a generalization of arrhythmia detec- tors. Indeed, PVC morphology changes from a subject to another and from a situation (at rest, say) to another situation (sport activity, say). In literature, complex meth- ods combining learning algorithms, clustering techniques, adaptive thresholds, morphology detection among others are reported. In this paper, a very simple method for PVC detection is described. This method is based on the use of a single threshold applied to the magnitudes of wavelet de- tail coefficients measured over ECG signals. The method is tested against records (106, 116, 119, 205 and 221) of the MIT-BIH Arrhythmia database (MITDB) [4]. These records were chosen because they contain normal QRS beats (N) and PVC beats. Records 116 and 205 contain other kinds of arrhythmia which were not considered in this work. The results were satisfactory and prove the reliability of the proposed method. A generalization of the proposed method can hardly be done using the single threshold scheme but can be done combining the present work to other detectors reported in literature. This paper is organized as follows. In section 2, the method is explained in details. Results and discussions are made in section 3 and section 4 concludes this paper.

\subsection{MIT-BIH Arrhythmia Database (MITDB)}

\section{Method}

MIT-BIH arrhythmia database (MITDB) [4] is a widely used ECG database available freely in physionet website [5]. It contains 48 records of 30 min length each with

$360 \mathrm{~Hz}$ sampling frequency. The ECG records present a

variety of waveforms, artifacts, complex ventricular, junc- tional and supraventricular arrhythmias and conduction ab- normalities. Each record is accompanied by an annotation file in which each ECG beat has been identified by expert cardiologists. These labels are used to evaluate the perfor- mance of our detector. In this work we have chosen to use only five records $(106,116,119,205,221)$ which contain normal $(\mathrm{N})$ and PVC beats. Records 116 and 205 contain other kinds of arrhythmia which were not considered in this work.

\subsection{Discrete Wavelet Transform}

Discrete wavelet transform (DWT) decomposes a signal at different scales. The signal is passed through series of mirror filters (high pass and low pass filters). At each level, the high pass filter produces detail information $d_{n}$ while the low pass filter produces coarse approximations $A_{n}$. In DWT decomposition 
over level $\mathrm{j}$, all obtained de- tails are kept whereas only the coarse approximation ob- tained in level $\mathrm{j}$ is kept, i.e., for level $\mathrm{j}$ decomposition of a signal we will obtain $\mathrm{j}+1$ coefficients ( 3 detail coefficients

+1 coarse approximation) representing the decomposed signal. Several wavelet families exist and the choice of the appropriate wavelet is important. In this paper we are using HAAR wavelet (see subsection 2.3 for choice ex- planation).

\subsection{ECG Signal Decomposition}

In our previous work, we developed a QRS detector based on wavelet coefficients [6]. The detail coefficients ob- tained over level 5, used in [6] and shown in Table 1, show a great potential for QRS detection and also a great poten- tial of discrimination between normal $(\mathrm{N})$ and abnormal beats. This is the property we are using in this article to discriminate between normal beats $(\mathrm{N})$ and $\mathrm{PVC}$ beats.

Then, the same wavelet and same detail coefficients used in [6] are used in this paper. Table 1 shows corre-

Table 1. Correspondence between detail coefficients and range frequencies

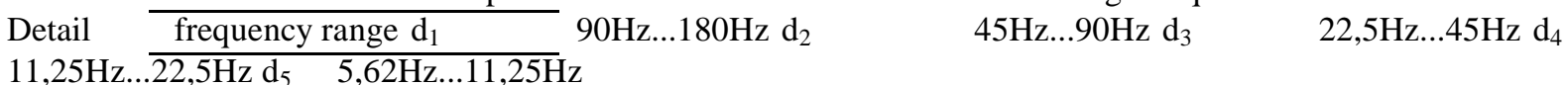

spondence between the details and range of frequencies. An example of ECG segment decomposed over level 5 using HAAR wavelet is shown in Figure 1. It is clear that there is a difference in detail coefficients magnitudes in the case of normal beats and PVC beats. Only detail co- efficient $\mathrm{d}_{5}$ shows comparable magnitudes for normal and PVC beats. Then, detail coefficients $d_{1}, d_{2}, d_{3}$ and $d_{4}$ can be used to descriminate between normal and PVC beats.

\subsection{Selection of appropriate detail coefficients}

Most of the energy of ECG signal is concentrated within the QRS complex [7]. Then, it is supposed that $\mathrm{d}_{3}, \mathrm{~d}_{4}$ and $\mathrm{d}_{5}$, which represent lowest frequencies (QRS complex en- ergy is withing low frequencies range) would be the most appropriate detail coefficients to be used to discriminate between normal and PVC beats. However, Figure 1 shows that $d_{5}$ can not be used to discriminate between normal and PVC beats as we can see the example of peaks cor- responding to normal and PVC beats depicted in Figure $1\left(\mathrm{~d}_{5}\right)$ where the magnitudes of both peaks corresponding to normal and PVC beats are similar. In contrary, according to Figure $1, \mathrm{~d}_{1}$ and $\mathrm{d}_{2}$ show a potential of discrimination. Evidently, more investigation is needed to decide which detail coefficients to use. Figures 2 and 3 show plots com- bining the different detail coefficients. In Figure 2, the magnitudes of $d_{3}$ corresponding to QRS beats are plotted versus magnitudes of $\mathrm{d}_{4}$ corresponding to the same QRS beats. It is clear that the two clusters (Normal and PVC) are somehow separated in the plan. Especially, clusters are better separated along $\mathrm{d}_{4}$ axis. Similarly, a plot (Fig- ure 3 ) of the magnitudes of $\mathrm{d}_{1}$ corresponding to QRS beats are plotted versus magnitudes of $\mathrm{d}_{2}$ corresponding to the same QRS beats. It appears from Figure 3 that the two clusters (Normal and PVC) are merged and their separa- tion using $\mathrm{d}_{1}$ and $\mathrm{d}_{2}$ is not possible. Then, we decided to use detail coefficients $d_{3}$ and $d_{4}$ to discriminate between normal and PVC beats. It appears also that the magnitudes of $\mathrm{d}_{3}$ and $\mathrm{d}_{4}$ corresponding to normal beats are higher than those corresponding to PVC beats. Then, the product of $\mathrm{d}_{3}$ and $\mathrm{d}_{4}$ is processed and used to discriminate between normal and PVC beats. The following algorithm summa- rizes the procedure used for PVC beats detection using Haar wavelet coefficients:

1 Apply Haar wavelet transform to ECG signal x(i), 


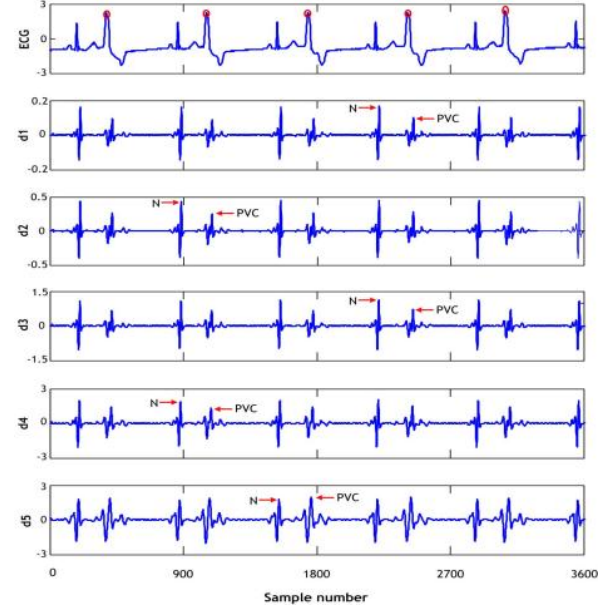

Fig. 1. Decomposition of ECG signal using Haar wavelet over level 5 for record 119 of the MITDB (segment in the range 22.2-45 s). ECG signal is depicted in $\mathrm{mV}$ whereas details are depicted in arbitrary units. PVC beats are marked by red circles. Example of peaks in $\mathrm{d}_{1}, \mathrm{~d}_{2}, \mathrm{~d}_{3}, \mathrm{~d}_{4}$ corresponding to normal and PVC beats are shown by red arrows.

3 PVC beats detection: Compare h(i) associated to QRS complex number i with a threshold $\lambda(0<\lambda<$ 1), if $\mathrm{h}(\mathrm{i})<\lambda \Rightarrow$ QRS number $\mathrm{i}$ is a PVC

beat else normal beat.

Figure 4 shows an example of the product $h$ applied to record 119 of the MITDB. In order to show the plot in 2D dimensions the magnitudes of $d_{3}$ corresponding to QRS beats are plotted versus values of $h$ corresponding to the same QRS beats. When screening Figure 4, we find that the two clusters (normal and PVC beats) are completely separated using the axis $h$ which means that for threshold $\lambda$ value in the range $0.47<\lambda<0.74$ all QRS beats are correctly classified. This is not the case for all records, Figure 5 shows an example of the magnitudes of $\mathrm{d}_{3}$ cor- responding to QRS beats plotted versus values of $\mathrm{h}$ corre- sponding to the same QRS beats in the case of record 106 which is not a completely separable case. Even though, the two clusters (normal and PVC beats) in the case of record 106 are not completely separable but the Figure 5 shows that the use of $h$ enhanced the separability in com- parison to Figure 3 where the two clusters are merged. Then, the choice of the value of $\lambda$ will influence the ob-

2 Compute the product $\mathrm{h}=\mid \Pi_{\mathbf{j}=3}^{4}$

are normalized),

$\mathrm{d}_{\mathbf{j}} \mid\left(\mathrm{d}_{3}, \mathrm{~d}_{4}\right.$ and $\mathrm{h}$

tained results. According to the obtained figures the value

$\lambda=0.45$ appears to be a good choice.

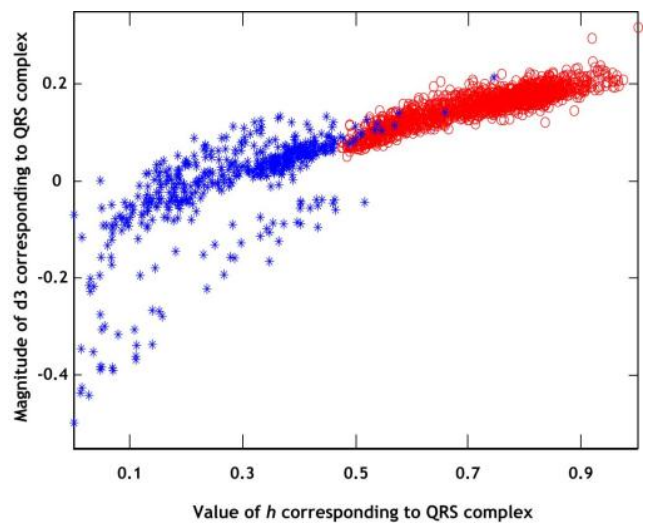

Fig. 2. Magnitudes of $d_{3}$ corresponding to QRS com- plexes vs. Magnitudes of $d_{4}$ corresponding to the same QRS complexes for the record 106 of the MITDB. $\mathrm{d}_{3}$ and $\mathrm{d}_{4}$ are depicted in arbitrary units. Normal beats are repre- sented by red circles whereas PVC beats are represented by blue asterisks. 


\section{Results And Discussion}

In order to evaluate the method developed in this paper we introduce three statistical measures. The sensitivity $\mathrm{S}_{\mathrm{e}}$, specificity $\mathrm{S}_{\mathrm{P}}$ and total classification accuracy $\mathrm{A}_{\mathrm{cc}}$ de- fined respectively as:

$\mathrm{S}_{\mathrm{e}}=$

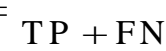

$\mathrm{S}_{\mathrm{P}}=\mathrm{TN}+\mathrm{FP}$
$\mathrm{TP}$

$\mathrm{T} \mathrm{N}$
$\mathrm{A}=$
$\mathrm{TP}+\mathrm{TN}$

$$
\mathrm{TP}+\mathrm{TN}+\mathrm{FP}+\mathrm{FN}
$$

where T P are the correctly classified PVC beats (True Positive), FP are misclassified normal beats (False Positive) which means normal beat classified as PVC beat,

FN are the misclassified PVC beats (False Negative) which means PVC beats classified as normal beats and TN are the correctly classified normal beats (True Negative). The sensitivity is used for evaluating the ability of the algo- rithm to detect PVC beats and the specificity is used for evaluating the ability of the algorithm to detect normal beats. The total classification accuracy $\left(\mathrm{A}_{\mathrm{cc}}\right)$ is used for evaluating the accuracy of the algorithm. These statistical measures are expressed in percentages.

Obtained results are summarized in Table 2. The proposed method performs well since total accuracy is $98.48 \%$ which is a good result. Actually, in this paper we showed that us- ing a single fixed threshold, one can obtain high classifica- tion accuracy of normal and PVC beats. This means that the chosen value $\mathrm{h}$ to characterize ECG beats and make the discrimination between two classes: normal and PVC

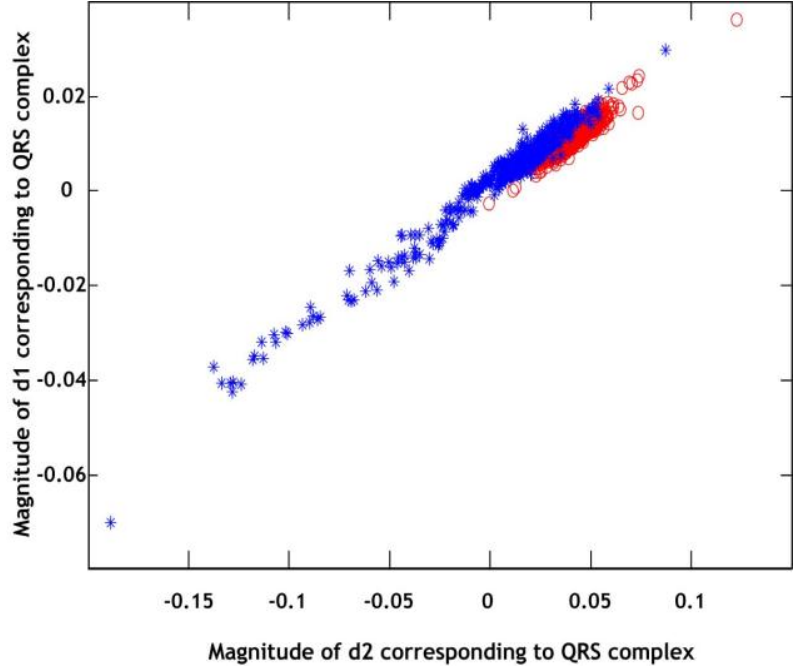

Fig. 3. Magnitudes of $d_{1}$ corresponding to QRS com- plexes vs. Magnitudes of $d_{2}$ corresponding to the same QRS complexes for the record 106 of the MITDB. $d_{1}$ and $d_{2}$ are depicted in arbitrary units. Normal beats are repre- sented by red circles whereas PVC beats are represented by blue asterisks.

beats is a good discrimination feature. This confirm our assumptions about the use of detail coefficients $\mathrm{d}_{3}$ and $\mathrm{d}_{4}$ which are in the frequency range of QRS beats. Based on the obtained results, we enumerate the most important findings and comments:

1. The proposed DWT based-method provides good results. Then, it appears that wavelet transform is well suited for ECG signal processing.

2. Generalization of the proposed method using a sin- gle fixed threshold to discriminate between the two classes: normal and PVC beats is not possible since the high inter-patient variations. However, the use of the value $h$ as a feature combined to other fea- tures for training/validation scheme, will enhance significantly obtained results.

3. Detail coefficient $\mathrm{d}_{4}$ is the best coefficient detail for characterizing and discriminating normal and PVC beats. 
4. DWT detail coefficients can also be used to detect other kinds of arrhythmias.

\section{Conclusion}

In this paper, a method to discriminate between normal and PVC beats was proposed. The method based on the use of DWT detail coefficients, uses a single fixed thresh- old to separate normal beats from the PVC beats. This threshold was obtained by combining two DWT detail co- efficients. Results are satisfactory and show that it is pos- sible to make good classification of normal and PVC beats using DWT detail coefficients. In order to generalize the

Table 2. Obtained results

\begin{tabular}{ccccccccccc} 
Records & Normal beats & PVC beats & TN & TP & FN & FP & $S_{\mathrm{e}}$ & $S_{P}$ & $A_{c c}$ \\
\hline 106 & 1507 & 520 & 1507 & 486 & 34 & 0 & 93.46 & 100 & 98.32 \\
116 & 2302 & 109 & 2244 & 107 & 2 & 58 & 98.17 & 97.48 & 97.51 \\
119 & 1543 & 444 & 1543 & 441 & 3 & 0 & 99.32 & 100 & 99.85 \\
205 & 2571 & 71 & 2570 & 69 & 2 & 1 & 97.18 & 99.96 & 99.89 \\
221 & 2031 & 396 & 1958 & 394 & 2 & 73 & 99.5 & 96.41 & 96.91 \\
Total & 9954 & 1540 & 9822 & 1497 & 43 & 132 & 97.21 & 98.67 & 98.48
\end{tabular}

proposed methods we need to calculate other features us- ing different methods such as methods based on ECG mor- phology. Then, combine these features to the feature de- veloped in this work and use them in a training/validation scheme using an optimal classifier such as support vector machine.

\section{References}

[1] O. Sayadi, M. B. Shamsollahi, G. D. Clifford, Ro- bust Detection of Premature Ventricular Contractions Using a Wave-Based Bayesian Framework, IEEE. T. BIO-MED. ENG. 57(2)(2010) 353-362.

[2] M. L. Talbi, A. Charef, PVC discrimination using the QRS power spectrum and self-organizing maps, Com- put. Method. Program. Biomed.94(3)(2009) 223-231.

[3] Y. Sun, K. L. Chan, S. M. Krishnan, Life-threatening ventricular arrhythmia recognition by nonlinear de- scriptor, BIOMED. ENG. ONLINE. 4(6)(2005) doi: 10.1186/1475-925X-4-6.

[4] G. B. Moody, R. G. Mark, The impact of the MIT- BIH Arrhythmia Database, IEEE. Eng. Med. Biol.20(3)(2001)45-50.

[5] R. G. Mark, G. $\quad$ B. Moody, MIT-BIH $\quad$ arrhythmia http://www.physionet.org/physiobank/database/mitdb/, accessed: 17 December 2012.

[6] Z. Zidelmal, A. Amirou, M. Adnane, A. Belouchrani, QRS detection based on wavelet coefficients, Com- put. Method. Program. Biomed. 107(3)(2012) 490-496.

[7] N. V. Thakor, J. G. Webster, W. J. Tompkins, Estima- tion of the QRS complex power spectra for design of a QRS filter, IEEE Trans. Biomed. Eng. 31(11)(1984)702-706. 